\title{
Navigation and motion control techniques for surface unmanned vehicle and autonomous ground vehicle: a review
}

\author{
Putri Nur Farhanah Mohd Shamsuddin ${ }^{1}$, Roshahliza M. Ramli ${ }^{2}$, Muhamad Arifpin Mansor ${ }^{3}$ \\ ${ }^{1,3}$ Faculty of Manufacturing and Mechatronic Engineering Technology, Universiti Malaysia Pahang, Malaysia \\ ${ }^{2}$ Faculty of Electrical and Electronic Engineering Technology, Universiti Malaysia Pahang, Malaysia
}

\section{Article Info \\ Article history: \\ Received Nov 2, 2020 \\ Revised Mar 2, 2021 \\ Accepted May 24, 2021 \\ Keywords: \\ Autonomous ground vehicle Motion control technique \\ Path following \\ Trajectory tracking \\ Unmanned surface vehicle}

\begin{abstract}
An excellent navigation, guidance, and control (NGC) system had a high impact on trajectory tracking and the following scenarios. Both scenarios will include the heading, tangent, and velocity parameters in the computation. However, the control system design problem is not a new issue in the unmanned surface vehicle (USV) and autonomous ground vehivle (AGV) due to this constraint faced by many researchers since early these autonomy developments. Hence, this paper listed and emphasizing the techniques, including techniques implementation, strength, and the algorithm's constraints, a fusion of several techniques implemented for vehicle's stability, a turning ahead, and heading estimation. This paper concerns the similar algorithm used in the USV and AGV. Most of the selected techniques are basic algorithms and have been frequently implemented to control both vehicles' systems. Previous research shows pure pursuit guidance is the most popular technique in AGV to control the degree-of-freedom (DOF) velocity and the dynamic rate (sway, surge, and yaw). Simultaneously, the line of sight (LOS) controller is very compatible with controlling the movement of the USV. In conclusion, the technique's simulation test needs further research that will expose in the actual situation.
\end{abstract}

This is an open access article under the CC BY-SA license.

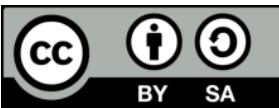

\section{Corresponding Author:}

Roshahliza M. Ramli

Faculty of Electrical and Electronic Engineering Technology

College of Engineering Technology

Universiti Malaysia Pahang

26600, Pekan, Malaysia

Email: roshahliza@ump.edu.my

\section{INTRODUCTION}

An autonomous vehicle is an advanced mechanism that operates without human intervention and decreases its risk from performing the mission. Based on the previous research, many unmanned surface vehicle (USV) and autonomous ground vehicle (AGV) performed longer in hazardous situations, such as; military identification, home protection, low-water surveys, and environmental surveillance [1]. Hence, autonomous vehicles' employment, which is consistently exposed to the risk situation, needs improvement in sensing, communication, and computing technologies. The USV and AGV consist of four types of mechanism which depends about the mission. For example, AGV are used for operation on the land, USV for water mission, unmanned underwater vehicle (UUV) for underwater surveillance, and unmanned aerial vehicle (UAV) for air operation. All these mechanisms communicate with the operator for several systems and link them together to perform the task. For example, UUV was built a relay interaction network on the water to develop wireless communication with the USV [2], [3]. In 2017, the latter was deployed in USV that built the communication with the REMUS100 AUV by using an automated method that involved movement 
between them [4]. This paper concerning two types of vehicles, USV and AGV, explores these vehicles' performance based on similar techniques implemented in the control system and how their performances are based on the techniques.

Several primary automation vehicles have researched smart USV and AGV and made technological progress in producing them [5]. An automated vehicle system's autonomy generally has differed into six levels, as defined in SAE-J3016 [6]. Besides, listed that six components were divided into the USV and AGV's vital components: the onboard system comprising additional structural elements, propulsion power system embedded, data gathering equipment, navigation, and control systems (GCC), ground station, and the system of communication. Stated the onboard system complementarily entails the guidance, navigation, and control subsystem, which work in interaction with each other [7]. Besides, the NGC module requires onboard software installation instruction through the computer to manage the entire USV and AGV. The navigation system comprehensively assists USV and AGV in identifying the current and future pose between two positions that are examined based on sensing, state estimation, environment perception, and situation awareness. The standard navigational devices have been introduced, such as global positioning system (GPS), electronic compass, inertial measuring unit, and gyroscope. The additional module consisting of IMU and stereo camera was designed to increase the robustness to decide if other sensors operate.

The USV and AGV are ordinarily designed based on the sizeable power-to-weight proportion. Due to these properties, new motion control concepts that involve the motion and the speed of the USV and AGV are highly demanded in this area of autonomy. However, motion control is the biggest problem that occurred in USV and AGVs. Previous studies have reported that the control system design problem is not a new issue in the USV and AGV due to this constraint faced by many researchers since the early autonomy development by upgrading several techniques to enhance the motion control system. In 2010, Oh and Sun stated the excellent control system had a high impact on the trajectory tracking and the path following scenarios. Hence, the control community studies attracted many researchers to upgrade the onboard subsystem [7].

The vessel's movement will depend on its transfer function, which is defined and measured in the DOF state. The vessel's transfer function used the term response amplitude operator (RAO) collectively for associated the phase angles, which respect the movement of the USV's centre of gravity. A computation of AGV and USV displacements, acceleration, and velocity will be calculated by RAO and generally issued in the following format for each wave period and direction in Table 1.

Table 1. Vessel six degrees of freedom

\begin{tabular}{ccc}
\hline Wave Period and Direction & Transfer Function & Phase Angle \\
\hline Surge $(\mathrm{m} / \mathrm{m})$ & $\mathrm{X}$ & $a_{x}$ \\
Sway $(\mathrm{m} / \mathrm{m})$ & $\mathrm{y}$ & $a_{y}$ \\
Heave $(\mathrm{m} / \mathrm{m})$ & $\mathrm{z}$ & $a_{z}$ \\
Roll $(\mathrm{deg} / \mathrm{m})$ & $\mathrm{R}$ & $a_{R}$ \\
Pitch $(\mathrm{deg} / \mathrm{m})$ & $\mathrm{P}$ & $a_{P}$ \\
Yaw $(\mathrm{deg} / \mathrm{m})$ & $\mathrm{Y}$ & $a_{Y}$ \\
\hline
\end{tabular}

The guidance system generates optimal trajectory commands that are smooth and feasible to help USV and AGV move towards the next position. All the commands are continuously updated according to the information provided from other medium platforms such as vehicle capability and navigation systems. The navigation system defines current and potential AGV and USV through location, orientation, speed, and acceleration. Information about the surrounding environment was included when the USV and AGV current and past positions are determined [8]. This method needs to deploy several sensors to estimate the current situation. Generating a good control system to identify the appropriate control forces and moments will produce a navigation and guidance system that had built the same trajectory with the target. Several kinds of research discussed the USV motion control was designed theoretically based on the AGV [9]. The waypoint navigation and remote-control mode are interfaces in the USV motion control to enhance the functionality of this autonomy [10]. Increase USV performance for the high-exactness mission, which concentrates in shallow water by using traditional autopilot structure in the guiding controller of the AGV [11]. By adding a GPS in the autonomous onboard system, the conventional motion control technology can dignify the autospeed, straight-line, and auto-heading towards the target position.

Figure 1 and Figure 2 show the AGV and USV vehicle's longitudinal (x), lateral (y), and vertical (z) axis based on the linear and rotational movement. Surge, sway, and the heave reckons as linear movement, while roll, pitch, and yaw are known as rotational movement. The vessel's movement will respect the surge, sway, heave, roll, pitch, and yaw, known as six degrees of freedom (DOF). 


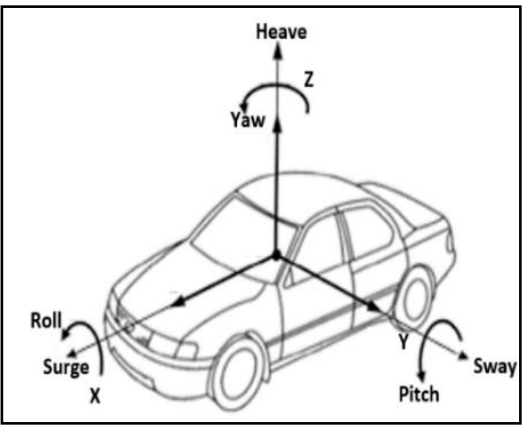

Figure 1. AGV's Six DOF

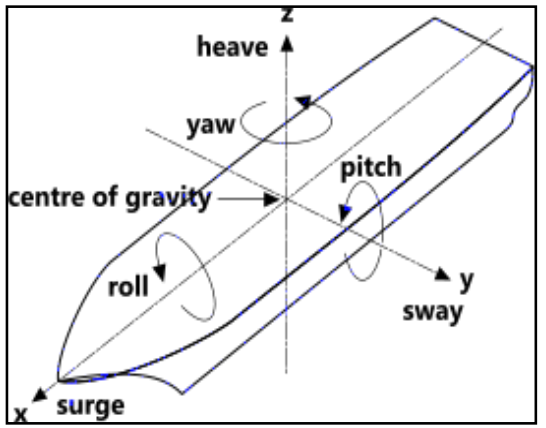

Figure 2. USV's Six DOF

The design of USV and AGV generates the reference curve for the path based on the time parameter called trajectory tracking. In-depth, Amer et al. [12] stated that this scenario refers to the AGV characterized by the motion's direction preferred by the control design. The control system focuses more on practical implementation on conserving the reference speed and reducing time limitations. For example, the constrained reinforcement learning concept helped decrease $84 \%$ waiting for the vehicles' time optimization and constant improvement of signal and trajectory for USV and AGV operation modules [13]. Nevertheless, this paper does not deal with neural network method studies.

A path-following algorithm is a crucial part of motion control by defining the distance, computing the trajectory path, and hitting the waypoint position by reducing the distance to zero. These scenarios will include the heading, tangent, and velocity parameter in the circumstance. For example, the mother vessel will follow the trajectory path design in the control system. Then, the controller will be generating the path that estimating the distance with the mother vessel.

Figure 3 shows the vehicle's kinematic equation describing the velocity in global coordinates, $v_{X}, v_{Y}$, and local coordinates, $\mathrm{v}_{\mathrm{x}}$, and $\mathrm{v}_{\mathrm{y}}$. The total velocity, $v$, and the vehicle's heading, $\theta$, concern the local coordinates while the vehicle's orientation, $\psi$, is more intended on the global coordinates. Figure 4 shows the illustration of the USV position follows the trajectory path after estimate the heading error. The USV heading $\theta_{\mathrm{d}}$ examines the waypoint position to identify the heading error based on both platforms. The turning radius, $\delta$ of the propellers, depends on the estimation radius, $r$ on the cartesian plane. USV turns to the correct angle based on the USV displacement, v. Based on the figure, the dotted line refers to the USV platform's motion while the entire line refers to the current position of the USV. The USV movement is depending on the commands generated by the controller.

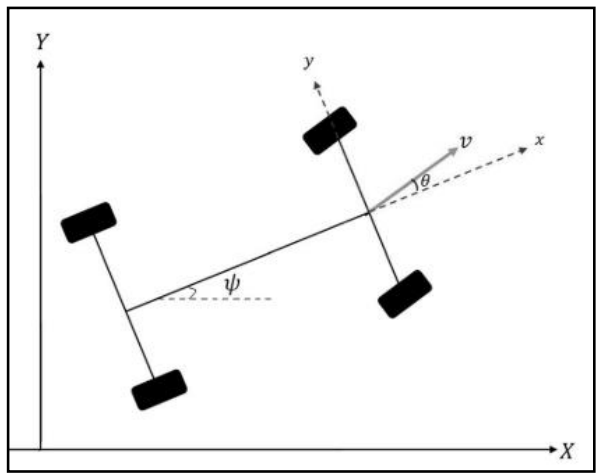

Figure 3. AGV in kinematic model towards desire heading [12]

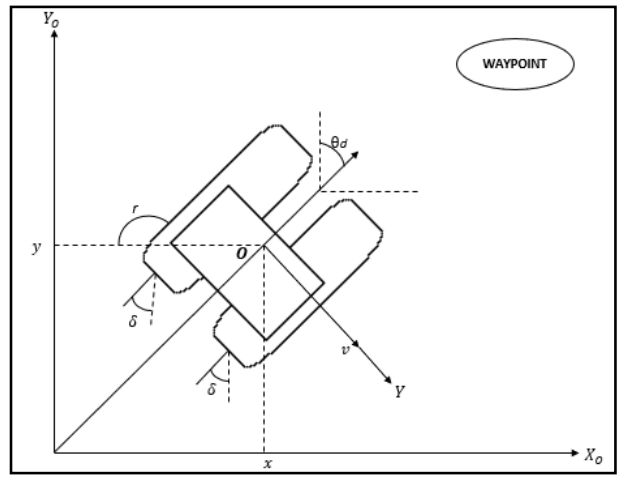

Figure 4. USV in kinematic model towards desire heading

In mechanical studies, kinematic is characterized as observing a body's motion without regard to its internal energy, forces, and inertia. Consequently, in terms of position, speed, and acceleration, the cinematic vehicle model can represent movement, regardless of internal dynamics. Modelling for kinematic vehicles can be used in most monitoring studies because of its simplicity and essential relationship to its motion. This 
model is commonly used to describe the multiple vehicular velocity and acceleration in the lateral direction and the vehicle's yaw motion with local coordinates and global coordinates [13].

The path that is generally known as the waypoint is a series of discrete points generating a command by the controller to reach the next position. Target speed is used as a reference point to produce a waypoint represented by $\mathrm{x}, \mathrm{y}$, direction, and velocity to determine a two-dimensional space's location and direction. In 2003, Petrinec et al. [14] investigated the AGV and USV that move on the path, defined as a straight line between two waypoints. Designing a good control law will increase the performance of the USV and AGV. However, to reach a time parameterized, USV and AGV control law needs to follow the reference trajectory's motion, which, also known as the common problem, occurred in typical motion control. Trajectory tracking controller design is more accessible deployed in an actuated vehicle, but under-actuated controller design unable to fully feedback linearized due to the non-holonomic constraints was occurred [15].

Based on the review of several journals and conference papers, the author concerns the similar algorithm used in the USV and AGV. Most of the selected techniques are basic algorithms and have been frequently implemented to control both vehicles' systems. The techniques selected are based on the prevalent method implemented in the autonomous vehicle's control system since 2010, which included: pure pursuit (PP) guidance, line of sight (LOS) guidance, constant bearing (CB) guidance, Lyapunov-based guidance, kalman filtering, backstepping method, predictive trajectory planning, and motion goal prediction.

\section{TECHNIQUES IMPLEMENTATION}

This section lists the selected techniques based on the papers reviewed and chronologically arranged from the early implementation until the latest outcome. This sub-topic will describe the techniques, including techniques implementation, strength, and the algorithm's constraints, a fusion of several techniques implemented for vehicle's stability, a turning ahead, and heading estimation. The authors discrete and discuss the most popular and rarely used technique within USV and AGV in section 3.

\subsection{Line of sight guidance}

LOS guidance is the missile method used in designing the reference heading and the straight-line direction for the AGV and USV control system. Commonly, the LOS algorithm combines with other practical techniques for generating the new method that enhances vehicle trajectory performance. Qiaomei et al. [16] stated that tracking control for the ship using the LOS algorithm could utilize the waypoint guidance algorithm in the simulation. In 2016, Bibuli et al. [17] propose integral LOS (ILOS) to increase the robustness concerning external and environmental disturbance. However, the ILOS method will be interrupted by the high lateral gain that causes unstable behaviour. Several studies implement the LOS method in AGV and USV autonomy, such as [18]-[21].

In the same vein, Charajia et al. [22] proposed the path following an algorithm that deployed the ILOS method in the underactuated USV in the presence of the environmental disturbance. The simulation of the parameter control design results in accurate stability of the global asymptotic stability (UGAS) as well as of the same local exponential stability (ULES). The ILOS controller can also track the path with disturbance by combining it with other algorithms [23]. The LOS technique had associated and obeyed the COLREGs rule to prevent the avoidance of other civilian vessels and obstacles.

In 2016, Moe and Pattersen [24] suggested LOS guidance to estimate the moving obstacles' trajectory path and ensure that the USV abides by the COLREGs while following the predefined desired path. Furthermore, adaptive LOS (ALOS) is introduced in the control system design to approximate the small-angle linearization. Both the ILOS and ALOS schemes are concentrating on managing the timeinvariant sideslip angle. In contrast, Due to external disturbances, or when the AGV and USV tracks a curved path, the sideslip's angle is often time varying.

A guidance law for ALOS is proposed to establish the straight and curve paths via the Fuzzy Logic optimization algorithm. For example, Mu et al. [25] introduced a fuzzy logic optimization in the ALOS to optimize the velocity or heading value between the path. Based on the simulation and theoretical analysis, the effectiveness and correctness are shown in the whole path following strategy. Previous studies have combined other LOS guidance methods to achieve the task's requirement in the control system [26]-[32].

\subsection{Pure pursuit guidance}

Another popular method to update the vehicle position and path commands is PP guidance. This approach is a geometric controller that uses simple and fewer parameters. In 2017, Ameer et al. [33] published a paper that describes the geometric controller as suitable for research that eliminates the velocity and acceleration response. However, PP guidance needs to affiliate with other methods to enhance the vehicle's internal force commands. Besides, the deployment of PP guidance is usually found in the AGV 
instead of other autonomy [34], [35]. Several autonomies also had implemented PP guidance in the control system for better performance in turning towards the target.

For example, the PP controller computes command in autonomous control design to reach a specific location or to follow in the case of a moving waypoint. The PP controller's trajectory path commanded the AGV and USV to maintain their pose over the waypoint, called dynamic positioning [36]. PP guidance can maintain a vehicle's position on the path in the curvature motion with high speed. This method also tends to chase the target in a short period. In 2008, Breveik et al. [37] examined the PP guidance to move toward the target with high speed. Previous studies have also deployed the PP guidance in the control design for curvature and turning path commands [38]-[42]. In the year 2000, PP guidance was implemented in the sizeable AGV and USV control design for estimating vehicle position and generate the steering commands [43].

PP guidance examines the look-ahead distance for designing the virtual path to creating comments in the control system [44]. By computing both positions, the PP guidance controller examines the setpoint and the vehicle's pose. PP guidance contributes to many improvements in the control system to enhance performance and eliminate computational complexity [45]. However, PP guidance in several AGV and USV are implemented because the decency in execution occurred. Implementation of a PID controller with PP guidance has a constraint to optimize and overshoot the parameter. PP guidance usually enhances the AGV's controller's stability despite it founded in the USV implementation [46].

\subsection{Constant bearing guidance}

In angle estimation and the USV and AGV's velocity is the prevailing method for CB guidance. The deployment of $\mathrm{CB}$ guidance in the control design increases the number of researchers to explore the capability of the USV in communication with other autonomy. Breivik and Loberg proposed control design research to improve the underway docking application for USV [47]. In 2015, Agrawal and Dolan demonstrated the deployment of CB guidance in the dynamic environment [48].

This algorithm is crucial to prevent USV collides with a nearby marine vessel, harbour, or coastal region by following the maritime, called COLREGS (rules of the road) [48]. Based on previous studies, Investigating CB guidance is more founded in the underactuated USV instead of actuated USV. In 2015, Norgen implemented a CB guidance scheme for tracking the velocity and angle of AUV and estimate the position of this autonomy [49]. In the same vein, Breivik and Loberg [47] have used CB guidance in the underactuated USV for tracking the waypoint of the mothership.

The control system generates specific motion with the mother vessel based on the command to control the USV movement. CB, LOS, and PP guidance are examples of the missile community whereby the interceptor must adjust the relative interceptor-target velocity along the interceptor-target line of sight [50]. CB guidance is the parallel navigation, which is called a two-point guidance scheme when referring to the USV trajectory aligned with the current waypoint velocity [51]. The distance between USV and the mother vessel followed the virtual target command set based on the motion range.

Some research implements the CB guidance controller for avoiding the obstacles, either static or moving on the path. Bibuli et al. [51] stated that USV needs computation highly for matching the motion between the vehicle and the mother vessel because this scenario is known as target hazardous. Hence, the guidance will force USV to follow the new trajectory path, free from the obstacle and safe to manoeuvre towards the next waypoint [52]. Furthermore, CB guidance's target tracking behaviour has like PP guidance to catch the waypoint by matching the velocity with the target [53], [54].

\subsection{Lyapunov-based guidance}

Recently, in vitro studies have shown that the Lyapunov concept can control the stabilities of the USV by tracking the heading and estimate velocity in cluttered environments. Bai et al. [55] found that an input saturation was solved using Lyapunov guidance in designing the path following the controller. commonly, Lyapunov guidance is implemented with another algorithm to solve the problem in the control design. The backstepping method was design based on the Lyapunov concept to increase the stability of USV [55]. Also, Bibuli et al. [54] have implemented the Lyapunov concept in the integral of LOS guidance (ILOS) that advances closed-loop kinematic systems.

The slave vessel communicates with the mother vessel by implementing it to decrease an error and avoid the collision. Indeed, the slave vessel can maintain the mother vessel's distance by computes the velocity of the surge, yaw, and sway of the autonomy. The Lyapunov concept's implementation maintains the distance and computes the mother vessel's velocity and heading position either in a linear or curvilinear path. virtual target (VT) implements the Lyapunov concept, where the slave vehicle will aim the VT that was calculated in the control design. Previous research stated that the slave vessel control design is used the Lyapunov concept to generate a reference path to follow a virtual target approach [56].

The fundamental theorem in the Lyapunov established the USV and AGV based on the equilibrium point. However, the control system faced the complexity of decreasing the aberrance of the steering 
dynamics occurred. A converse theorem exists in the control system, interrupting the vehicle's balancing mode when USV and AGV equilibrium is stable. Hence, the function generated by the execution is affected due to computational constraints [57]. The Lyapunov controller can stabilize the USV and AGV's nonlinear system besides solving the setting control problem.

\subsection{Predictive trajectory planning}

Coast guard collision regulation (COLREGs) is the vessel's rule through high water traffic. Predictive Trajectory Planning was introduced in the onboard system to increase USV and AGV guidance. The vehicle's position and the target will be computed and formed the trajectory commands, which comprises collision-free. It will minimize the distance and collision that occurred between the vehicle. This model's structure implemented in the USV and AGV bears the same concept as the target approach [58]. For example, the algorithm was introduced to complies with the COLREGs among civilian vessels and the static obstacle [59]. A five-dimensional trajectory planner based on the lattice was implemented in the water high traffic operation [60].

Similarly, the USV control system implemented lattice-based trajectory planning to reach the moving target. The lattice-based model increases motion safety by guiding USV to follow the trajectory path in high traffic [61]. Besides, Liu et al. [62] develop the Kalman filter-based predictive planning model to enhance the performance of estimate the moving ship's trajectories and track the position of onboard USV in real-time. USV dynamics and controlling the action of primitives scales to minimize the search performance on the congestion on the specific region will also be implemented in the planner.

However, the speed of the USV and AGV became lower. It caused the trajectories is slightly offed from the original path when this vehicle is taken different thrusts within the tracking time. The tendency to lose the target is high when the computation program faced the limitation by estimating the target's distance. A range prediction set by the user is lower than the actual [63]. Based on previous research, the implementation of this Predictive Trajectory Planning gives an excellent performance in planning the target's path by combining with other methods [64]-[66].

\subsection{Kalman filtering}

The name kalman filtering comes from the tasks the method was designed. The USV and AGV need a good navigation system to enhance the performance of guidance. Many researchers utilized an inertial navigation system (INS) and the global navigation satellite system (GNSS) onboard vehicle because of the complementary nature between both electronic principles. Implementation of traditional kalman filtering (KF) able to estimate the position accurately. In 2012, Tran et al. [67] examined the KF method's capability in the attitude heading reference system (AHRF). AHRF gave an excellent performance in estimating the roll, pitch, and yaw angles. However, Wang et al. found using a traditional Kalman filter, which displays a more massive state error than usual due to the noisy data. Due to this problem, a five-by-one median filter has been added to eliminate the stereo matching error, which interrupted the data [68].

Furthermore, the KF method will cause the cascaded filtering problem due to the GPS data processing used the KF while the other method used in the integration. By employing centralized KF, this problem had been solved [69]. Extended kalman filter (EKF) undergoes the instructions by associating INS and GNSS data fusion. Furthermore, EKF triggers a nonlinear system that develops the commands in advance for creating a new trajectory. Several studies Implementing EKF have been carried out on [70]-[72]. However, the posterior mean and covariance calculation will produce a considerable error value when EKF is included in the computation [73]. The use of EKF still needs improvement for increasing navigation accuracy.

Some errors had been led in tracks the target and within processing the data. The fusion strategy was developed by employing iterated sigma point kalman filter (ISPKF) with interacting multiple model (IMM) to control kinematic and measurement models' changes. This paper concentrates on estimating an aerodynamic parameter and process the trajectory data. In 2015, Lecce et al. [74] analyzed the filtered data. The aerodynamic coefficients had been taken to compare with data obtained from the vehicle configuration that used the traditional UKF and ISPKF method. Subsequently, ISPKF ready to improve the precision and the vigour of the UKF. However, the implementation of the UKF still faced a constraint in eliminating the gaussian distribution.

\subsection{Backstepping method}

The backstepping method comprises an algorithm employed in many USV and AGV due to the capability to track and follow the setpoint based on the commands onboard had set up. Several studies give attention to this method for developing the under-actuated vessel [75]. Persistent exciting (PE) is an example of the backstepping-based model implemented on the USV and AGV to control the under-actuated USV and 
AGV. In-depth, PE potentially drives USV and AGVs on arbitrary trajectories, including circle, straight-line, and curved trajectories.

In 2011, Ma et al. [75] introduced the new straight-line path-following control algorithm for improving path following control performance. Combining two methods: yaw angle feedback and backstepping technique in the control design increases the closed-loop stability-besides, the straight-line path following algorithm shown the feasibility and validity in the simulation results. Furthermore, an adaptive dynamical sliding mode is an example of backstepping implementation to validate the performance of underactuated USV follows the trajectories [76]. In the same vein, Chen et al. [77] proposed research to control the movement and follow the trajectories based on the Backstepping method assisted with the adaptive mode control theory. The result has shown good performance in tracking the nonlinear USV and AGV control system.

In 2018, Deng et al. [78] designed a fusion of three techniques, Lyapunov, backstepping, and genetic algorithm (GA), for creating a right angle and speed tracking. Lyapunov-based stability theory increases the global asymptotic stability and maintains the USV and AGV heading to follow the trajectories. In 2017, Liu et al. [79] implemented sliding mode integration and dynamic neural method internally highprecision and fast navigation response that consist of the internal backstepping control loop to enhance the control system performance. Many researchers have also implemented the neural mode model to realize the USV and AGV's accurate and stable tracking in the steering process [80].

\subsection{Motion goal prediction}

Motion goal prediction is reckoned as a real-time planner when this algorithm computes the commands directly onto the onboard system to generate the trajectories. Three elementary processes will consult the algorithm's ultimate output, including the USV and AGV desired pose, the vehicle's velocity, and arrival time. The low-level controller executes the commands neatly, maintains the position on the track, and receives the target within the time. Hence, this algorithm can guide the USV and AGV in cluttered surroundings by controlling its onboard action. In 2014, Svec et al. [81] overcame the USV operation in the cluttered environment by implementing the motion goal prediction algorithm. The planner computed the distance between two vehicles and complies with the guiding commands to maintain the USV and AGV on the trajectories. This algorithm had designed for an un-predefined position because the vehicle might not have earlier data about the objective vessel's proposed movement.

\section{RESULTS AND DISCUSSION}

Table 2 lists the capabilities and advantages of the techniques used in the AGV and USV control systems. Among all the studies reviewed so far, stability control is the most critical for USV and AGV studies. Hence, the fusion of techniques is crucial in the USV and AGV to create the best control design. This is because the USV and AGV need many capabilities to move to achieve the task requirement by including all the aspects, especially the tracking performance. The fusion of LOS and the fuzzy logic method significantly impacts the vehicle stability for three significant controls; straight line, curve, and heading modification. Employment of the backstepping method with another technique creates a new AGV and USV control system that is more stable in terms of DOF control, state estimation of the path, and collision avoidance. However, a fusion of computation techniques will reduce the system's efficiency and generalize the commands quickly.

Several techniques implemented in the control system design were selected and analyzed based on the method's ability to perform the task. Based on the observation, most control system techniques are designed to manipulate the speed, heading position between the USV and AGV, and the target path. The LOS, CB, and PP guidance are the most suitable suggestions for reducing the tendency to lose the target. LOS, CB, and PP controllers' implementation usually enhance the performance of the USV and AGV motion topic. Previous research shows that the LOS controller is the most popular technique to control the DOF dynamic (sway, surge, and yaw) rate. Many researchers implemented the LOS controller in their experiment to control the DOF and follow the trajectory path because this controller is easily implemented on the USV. Several techniques were also associated with the LOS controller to set off the hydrodynamic state on the water surface during the following process. The effectiveness of controlling AGV and USV in the timeparameterized by the heading reference and the waypoint design will increase the LOS controller's employment.

PP guidance is a famous method in the AGV guidance performance of stability, turning ahead, and heading estimation. However, this controller is practised only in a few studies for the USV, such as done in [82], [83]. Next, the performance of AGV and USV for tracking the position will be promptly increased by using a fusion of Kalman filtering to generate the heading and eliminate the system's noise disturbance. Furthermore, motion goal prediction, Lyapunov, and the predictive planning model were implemented in the 
computation control system to enhance the vehicles' DOF's stability and increase the jumbled condition's robustness, such as high traffic the water surface. LOS, PP, and CB controller is known as the missile technique, which is technically designed for chasing the target during the duration set.

Table 2. The analysis of AGV and USV techniques

\begin{tabular}{|c|c|c|c|c|c|c|c|c|}
\hline Techniques & LOS & PP & $\mathrm{CB}$ & Lyapunov & Predictive Trajectory & KF & Backstepping & Motion Goal \\
\hline \multicolumn{9}{|c|}{ Stability Control } \\
\hline Straight-Line Path & ST & TT & TT & TT & - & TTT & TT & TT \\
\hline Curve Path & ST & TT & TT & TT & - & TTT & - & - \\
\hline Heading Modification & TTT & TT & TT & & TT & TTT & - & - \\
\hline Sideslip Angle & \multicolumn{8}{|c|}{ DOF Control } \\
\hline Yaw & ST & ST & ST & TT & - & TTT & ST & ST \\
\hline Sway & ST & ST & ST & - & - & TTT & ST & ST \\
\hline Surge & ST & ST & ST & - & TT & TTT & ST & ST \\
\hline \multicolumn{9}{|c|}{ State Estimation } \\
\hline Predefined Path & ST & TT & TT & ST & - & - & ST & - \\
\hline Unknown Position & - & TT & - & - & - & ST & - & TT \\
\hline \multicolumn{9}{|c|}{ Collision Avoidance } \\
\hline Static Obstacle & ST & TTT & - & - & TT & - & - & - \\
\hline Moving Obstacle & ST & TTT & - & ST & TT & TT & ST & - \\
\hline \multicolumn{9}{|c|}{ Motion Control } \\
\hline Constant & - & - & TT & - & TT & - & - & - \\
\hline Variation of Motion & TT & - & TT & - & TT & ST & - & TT \\
\hline \multicolumn{9}{|c|}{ Vehicle Actuation } \\
\hline Under Actuation & ST & TT & ST & ST & ST & ST & ST & ST \\
\hline Fully Actuation & - & - & - & TT & - & - & - & - \\
\hline
\end{tabular}

Note: ST for Standard Technique, TT for a fusion of Two Technique, TTT for fusion more Than Two Technique

\section{CONCLUSION}

In conclusion, this paper had reviewed more than 50 papers of literature from the last ten years about the techniques in AGV and USV developments. Path following and trajectory tracking techniques were emphasized in this paper to identify and validate each technique's functionalities for AGV and USV performance. The deployment of the technique was customized based on the requirement and the risk of the mission. LOS guidance was suggested in many research for tracking the heading angle and velocity of the target, which will guide the AGV and USV towards the position. Based on the analysis given, the fusion of Kalman filtering is the prevalent method for navigation purposes. It gives a good performance in the state estimation in the underactuated AGV and USV. The technique which was performed in the simulation test needs further research that will expose the actual situation. The motion goal prediction method is proposed to track the un-predefined target by designing the algorithm that concerns the desired heading angle and velocity parameter.

\section{ACKNOWLEDGEMENTS}

This research was financially supported by the Universiti Malaysia Pahang and the Automotive Excellent Centre under grant no RDU1903103.

\section{REFERENCES}

[1] J. E. Manley, "Unmanned surface vehicles, 15 years of development," OCEANS 2008, 2008, pp. 1-4, doi: 10.1109/OCEANS.2008.5152052.

[2] J. M. Larrazabal and M. S. Peñas, "Intelligent rudder control of the unmanned surface vessel," Applications with Expert Systems, vol. 55, pp. 106-117, 2016, doi: 10.1016/j.eswa.2016.01.057.

[3] T. Liu, Z. Dong, H. Du, L. Song and Y. Mao, "Path Following Control of the Underactuated USV Based on the Improved Line-Of-Sight Guidance" Polish Maritime Research, vol. 24, no. 1, pp. 3-11, 2017, doi: 10.1515/pomr2017-0001.

[4] S. Sohn, J. Oh, Y. Lee, D. Park and I. Oh, "Design of a Fuel-Cell-Powered Catamaran-Type Unmanned Surface Vehicle," in IEEE Journal of Oceanic Engineering, vol. 40, no. 2, pp. 388-396, April 2015, doi: 10.1109/JOE.2014.2315889.

[5] Insights, C.: 30 Corporations Working On USV and AGVs. https://www.cbinsights.com/blog/autonomousdriverless-vehicles-corporations-list/. Accessed 26th April 2016 (2016) 
[6] SAE-J3016: Taxonomy and Definitions for Terms Related to On-Road Motor Vehicle Automated Driving Systems. In: O.-R.A.V.S. (ed.) Committee (2014)

[7] S. R. Oh and J. Sun, "Path Following of Underactuated Marine Surface Vessels Using Line-Of-Sight Based Model Predictive Control," Ocean Engineering, vol. 37, no. 2-3, pp. 289-295, 2010, doi: 10.1016/j.oceaneng.2009.10.004.

[8] C. R. Sonnenburg and C. A. Woolsey, "Modeling, Identification, and Control of an Unmanned Surface Vehicle" Journal of Field Robotics, vol. 30, no. 3, pp. 371-398, 2013, doi: 10.1002/rob.21452.

[9] J. Ebken, M. Bruch, J. Lum, "Applying unmanned ground vehicle technologies to unmanned surface vehicles" in Proceedings of SPIE 5804: Unmanned Ground Vehicle Technology VII, Orlando, Florida, the USA. 2005, vol. 5904, pp. 585-596, doi: 10.1117/12.605254.

[10] J. Majohr and T. Buch, "Modelling, Simulation and Control of an Autonomous Surface Marine Vehicle for Surveying Applications Measuring Dolphin MESSIN", IEE Control Engineering Series, vol. 69, p. 329, 2006.

[11] M. Caccia, M. Bibuli, R. Bono, and G. Bruzzone, "Basic navigation, guidance and control of an unmanned surface vehicle," Autonomous Robots, vol. 25, no. 4, pp. 349-365, 2008, doi: 10.1007/s10514-008-9100-0.

[12] N. H. Amer, H. Zamzuri, K. Hudha, and Z. A. Kadir, "Modelling and Control Strategies in Path Tracking Control for Autonomous Ground Vehicles: A Review of State of the Art and Challenges" Journal of Intelligent \& Robotic Systems, vol. 86, no. 2, pp. 225-254, 2017, doi: 10.1007/s10846-016-0442-0.

[13] Chiranjib Sur, "UCRLF: Unified constrained reinforcement learning framework for phase-aware architectures for USV and AGV signaling and trajectory optimization," Evolutionary Intelligence, vol. 12, no. (4), pp. 689-712, 2019, doi: 10.1007/s12065-019-00278-7.

[14] K. Petrinec, Z. Kovacic and A. Marozin, "Simulator of multi-AGV robotic industrial environments," IEEE International Conference on Industrial Technology, 2003, 2003, pp. 979-983 Vol.2, doi: 10.1109/ICIT.2003.1290794.

[15] G. E. Mullins and S. K. Gupta, "Adversarial blocking techniques for autonomous surface vehicles using modelpredictive motion goal computation," 2015 IEEE/RSJ International Conference on Intelligent Robots and Systems (IROS), 2015, pp. 2272-2278, doi: 10.1109/IROS.2015.7353682.

[16] S. Qiaomei, R. Guang, Y. Jin and Q. Xiaowei, "Autopilot Design for Unmanned Surface Vehicle Tracking Control," 2011 Third International Conference on Measuring Technology and Mechatronics Automation, 2011, pp. 610-613, doi: 10.1109/ICMTMA.2011.155.

[17] M. Bibuli, W. Caharija, K. Y. Pettersen, G. Bruzzone, M. Caccia, and E. Zereik "ILOS Guidance - Experiments and Tuning" IFAC Proceedings Volumes, vol. 47, no. 3, pp. 4209-4214, 2014, doi: 10.3182/20140824-6-za1003.01622.

[18] K. Hui, D. Phillips and A. Kekirigoda, "Beyond Line-of-Sight range extension with OPAL using autonomous unmanned aerial vehicles," MILCOM 2017 - 2017 IEEE Military Communications Conference (MILCOM), 2017, pp. 279-284, doi: 10.1109/MILCOM.2017.8170774.

[19] Z. Peng and J. Wang, "Output-Feedback Path-Following Control of Autonomous Underwater Vehicles Based on an Extended State Observer and Projection Neural Networks," in IEEE Transactions on Systems, Man, and Cybernetics: Systems, vol. 48, no. 4, pp. 535-544, April 2018, doi: 10.1109/TSMC.2017.2697447.

[20] S. Jung, S. Hwang, H. Shin and D. H. Shim, "Perception, Guidance, and Navigation for Indoor Autonomous Drone Racing Using Deep Learning," in IEEE Robotics and Automation Letters, vol. 3, no. 3, pp. 2539-2544, July 2018, doi: 10.1109/LRA.2018.2808368.

[21] J. Miao, S. Wang. M. M. Tomovic, and Z. Zhao, "Compound line-of-sight nonlinear path following control of underactuated marine vehicles exposed to wind, waves, and ocean currents," Nonlinear Dynamics, vol. 89, pp. 2441-2459, 2017, doi: 10.1007/s11071-017-3596-9.

[22] W. Caharija et al., "Integral Line-of-Sight Guidance and Control of Underactuated Marine Vehicles: Theory, Simulations, and Experiments," in IEEE Transactions on Control Systems Technology, vol. 24, no. 5, pp. 16231642, Sept. 2016, doi: 10.1109/TCST.2015.2504838.

[23] T. Liu, Z. Dong, H. Du, L. Song, and Y. Mao, "Path Following Control of the Underactuated USV Based on the Improved Line-of-Sight Guidance Algorithm" Polish Maritime Research, vol. 24, no. 1, pp. 3-11, 2017, doi: 10.1515/pomr-2017-0001.

[24] S. Moe, K. Y. Pettersen, T. I. Fossen and J. T. Gravdahl, "Line-of-sight curved path following for underactuated USVs and AUVs in the horizontal plane under the influence of ocean currents," 2016 24th Mediterranean Conference on Control and Automation (MED), 2016, pp. 38-45, doi: 10.1109/MED.2016.7536018.

[25] D. Mu, G. Wang, Y. Fan, Y. Bai, and Y. Zhao, "Fuzzy-Based Optimal Adaptive Line-of-Sight Path Following for Underactuated Unmanned Surface Vehicle with Uncertainties and Time-Varying Disturbances," Mathematical Problems in Engineering, vol. 6, pp. 1-12, 2018, doi: 10.1155/2018/7512606.

[26] N. Wang, Z. Sun, Z. Zheng, H. Zhao, "Finite-Time Sideslip Observer-Based Adaptive Fuzzy Path-Following Control of Underactuated Marine Vehicles with Time-Varying Large Sideslip," International Journal of Fuzzy Systems, vol. 20, no. 6, pp. 1767-1778, 2017, doi: 10.1007/s40815-017-0392-0.

[27] Z. Li, R. Bachmayer and A. Vardy, "Vector field path following control for unmanned surface vehicles," OCEANS 2017 - Aberdeen, 2017, pp. 1-9, doi: 10.1109/OCEANSE.2017.8084878.

[28] N. Tran, T. Nguyen, V. Tran, V. Nguyen and T. Nguyen, "The Design of an VIAM-USVI000 Unmanned Surface Vehicle for Environmental Monitoring Applications," 2018 4th International Conference on Green Technology and Sustainable Development (GTSD), 2018, pp. 33-37, doi: 10.1109/GTSD.2018.8595624.

[29] M. S. Wiig, K. Y. Pettersen, E. M. Ruud and T. R. Krogstad, "An Integral Line-of-Sight Guidance Law with a Speed-dependent Lookahead Distance," 2018 European Control Conference (ECC), 2018, pp. 1269-1276, doi: 10.23919/ECC.2018.8550243 
[30] M. Li, C. Guo and H. Yu, "Filtered Extended State Observer Based Line-of-Sight Guidance for Path Following of Unmanned Surface Vehicles With Unknown Dynamics and Disturbances," in IEEE Access, vol. 7, pp. 178401178412, 2019, doi: 10.1109/ACCESS.2019.2958855.

[31] H. Huang, Y. Li, Y. Zhuang, M. Gong, S. Sharma and D. Xu, "Line-of-Sight Path Following of an Underactuated USV Exposed to Ocean Currents using Cascaded Theorem," 2018 WRC Symposium on Advanced Robotics and Automation (WRC SARA), 2018, pp. 326-333, doi: 10.1109/WRC-SARA.2018.8584241.

[32] P. Huang, X. Luo, and Z. Zhang, "Headland Turning Control Method Simulation of Autonomous Agricultural Machine Based on Improved Pure Pursuit Model," Computer and Computing Technologies in Agriculture III IFIP Advances in Information and Communication Technology, pp. 176-184, 2010, doi: 10.1007/978-3-642-12220-0_27.

[33] A. Ratnoo, S. Y. Hayoun, A. Granot, and T. Shima, "Path Following Using Trajectory Shaping Guidance," Journal of Guidance, Control, and Dynamics, vol. 38, no. 1, pp. 106-116, 2015, doi: 10.2514/1. g000300.

[34] Y. Chen and J. J. Zhu, "Pure Pursuit Guidance for Car-Like Ground Vehicle Trajectory Tracking," in Proceedings of the ASME 2017 Dynamic Systems and Control Conference, Tysons, Virginia, USA, 2017, pp. 1-9, doi: $10.1115 / \mathrm{dscc} 2017-5376$.

[35] X. Wu, "Integrated Motion Control of Path Tracking and Servo Control for an Automated Guided Vehicle," Journal of Mechanical Engineering, vol. 47, no. 3, p. 43, 2011, doi: 10.3901/jme.2011.03.043.

[36] W. Wang, T. Hsu and T. Wu, "The improved pure pursuit algorithm for autonomous driving advanced system," 2017 IEEE 10th International Workshop on Computational Intelligence and Applications (IWCIA), 2017, pp. 3338, doi: 10.1109/IWCIA.2017.8203557.

[37] A. Gautam, P. B. Sujit, and S. Saripalli "Autonomous Quadrotor Landing Using Vision and Pursuit Guidance," IFAC-PapersOnLine, vol. 50, no. 1, pp. 10501-10506, 2017, doi: 10.1016/j.ifacol.2017.08.1982.

[38] X. Li, Z. Sun, D. Cao, D. Liu, and H. He, "Development of a new integrated local trajectory planning and tracking control framework for autonomous ground vehicles," Mechanical Systems and Signal Processing, vol. 87, pp. 118137, 2017, doi: 10.1016/j.ymssp.2015.10.021.

[39] K. Tanakitkorn, "A Review of Unmanned Surface Vehicle Development," Maritime Technology and Research, vol. 1, no. 1, pp. 2-8, 2018, doi: 10.331 75/mtr.2019.140730.

[40] M. Breivik and T. I. Fossen, "Guidance laws for planar motion control," 2008 47th IEEE Conference on Decision and Control, 2008, pp. 570-577, doi: 10.1109/CDC.2008.4739465.

[41] M. Park, S. Lee, and W. Han, "Development of Steering Control System for USV and AGV using Geometry based Path Tracking Algorithm," ETRI Journal, vol. 37, no. 3, pp. 617-625, 2015, doi: 10.4218/etrij.15.2314.0123.

[42] H. Niu, Y. Lu, A. Savvaris and A. Tsourdos, "Efficient path following algorithm for unmanned surface vehicle," OCEANS 2016 - Shanghai, 2016, pp. 1-7, doi: 10.1109/OCEANSAP.2016.7485430.

[43] A. R. Castaño, G. Heredia, and A. Ollero, "Analysis of a GPS-Based Fuzzy Supervised Path Tracking System for Large Unmanned Vehicles," in IFAC Intelligent Components and Instruments for Control Applications, Buenos Aires, Argentina, 2000, vol. 33, no. 25, pp. 125-130, doi: 10.1016/s1474-6670(17)39327-8.

[44] O. Amidi and C. E. Thorpe, "Integrated mobile robot control," Mobile Robots V, vol. 1388, pp. 504-523, 1990, doi: $10.1117 / 12.25494$.

[45] M. Breivik and T. I. Fossen, "Guidance Laws for Autonomous Underwater Vehicles," Underwater Vehicles, 2009, doi: $10.5772 / 6696$.

[46] P. N. F. B. Mohd Shamsuddin and M. A. B. Mansor, "Motion Control Algorithm for Path Following and Trajectory Tracking for Unmanned Surface Vehicle: A Review Paper," 2018 3rd International Conference on Control, Robotics and Cybernetics (CRC), 2018, pp. 73-77, doi: 10.1109/CRC.2018.00023.

[47] M. Breivik and J. E. Loberg, "A Virtual Target-Based Underway Docking Procedure for Unmanned Surface Vehicles," in Proceedings of the 18th World Congress the International Federation of Automatic Control, Milano, Italy, 2011, vol. 44, no. 1, pp. 13630-13635, doi: 10.3182/20110828-6-it-1002.02969.

[48] P. Agrawal and J. M. Dolan, "COLREGS-compliant target following for an Unmanned Surface Vehicle in dynamic environments," 2015 IEEE/RSJ International Conference on Intelligent Robots and Systems (IROS), 2015, pp. 1065-1070, doi: 10.1109/IROS.2015.7353502.

[49] P. Norgren, M. Ludvigsen, T. Ingebretsen and V. E. Hovstein, "Tracking and remote monitoring of an autonomous underwater vehicle using an unmanned surface vehicle in the Trondheim fjord," OCEANS 2015 - MTS/IEEE Washington, 2015, pp. 1-6, doi: 10.23919/OCEANS.2015.7401975.

[50] T. I. Fossen, M. Breivik, and R. Skjetne, "Line-of-sight path following of underactuated marine craft," in IFAC Maneuvering and Control of Marine Craft Proceedings Volumes, Girona, Spain, 2003, vol. 36, no. 21, pp. 211-216, doi: 10.1016/ s1474-6670(17)37809-6.

[51] T. I. Fossen, "The Brains of Motion Control Systems. Lecture presented at Guidance Systems," in Norwegian University of Science and Technology, John Wiley and Sons Ltd., 2011.

[52] M. Breivik, "Topics in Guided Motion Control of Marine Vehicles," Ph.D. thesis. Norwegian University of Science and Technology, Trondheim, Norway, 2010.

[53] M. Breivik and T. I. Fossen, "Guidance laws for planar motion control," 2008 47th IEEE Conference on Decision and Control, 2008, pp. 570-577, doi: 10.1109/CDC.2008.4739465.

[54] M. Bibuli, M. Caccia, L. Lapierre and G. Bruzzone, "Guidance of Unmanned Surface Vehicles: Experiments in Vehicle Following," in IEEE Robotics \& Automation Magazine, vol. 19, no. 3, pp. 92-102, Sept. 2012, doi: 10.1109/MRA.2011.2181784. 
[55] Y. Bai, Y. Zhao and T. Li, "The USV path following controller design," 2017 4th International Conference on Information, Cybernetics and Computational Social Systems (ICCSS), 2017, pp. 679-682, doi: 10.1109/ICCSS.2017.8091500.

[56] M. Bibuli, A. Gasparri, A. Priolo, G. Bruzzone, and M. Caccia, "Virtual Target based path-following Guidance System for Cooperative USV Swarms," in 9th IFAC Conference on Manoeuvring and Control of Marine Craft, Arenzano, Italy, 2012, vol. 45, no. 27, pp. 362-367, doi: 10.3182/20120919-3-IT2046. 00062.

[57] H. Qu, "Wind Feedforward Control of USV," Master Thesis, Florida Atlantic University, 2016.

[58] S. Campbell, W. Naeem, and G. W. Irwin, "A Review on Improving the Autonomy of Unmanned Surface Vehicles Through Intelligent Collision Avoidance Maneuvers," Annual Reviews in Control, vol. 36, no. 2, pp. 267-283, 2012 doi: 10.1016/j.arcontrol.2012.09.008.

[59] P. Svec et al., "Dynamics-aware target following for an autonomous surface vehicle operating under COLREGs in civilian traffic," 2013 IEEE/RSJ International Conference on Intelligent Robots and Systems, 2013, pp. 3871-3878, doi: 10.1109/IROS.2013.6696910.

[60] B. C. Shah, et al., "Resolution-adaptive risk-aware trajectory planning for surface vehicles operating in congested civilian traffic," Autonomous Robots, vol. 40, no. 7, pp. 1139-1163, 2015, doi: 10.1007/s10514-015-9529-x.

[61] P. Svec, A. Thakur, B. C. Shah, and S. K. Gupta, "USV Trajectory Planning for Time-Varying Motion Goals in an Environment with Obstacles," in Proceedings of the ASME 2012 International Design Engineering Technical Conferences and Computers and Information in Engineering Conference, 2012, vol. 45035, pp. 1297-1306, doi: 10.1115/DETC2012-71239.

[62] Y. Liu, W. Liu, R. Song, and R. Bucknall, "Predictive navigation of unmanned surface vehicles in a dynamic maritime environment when using the fast-marching method," International Journal of Adaptive Control and Signal Processing, vol. 31, no. 4, pp. 464-488, 2015, doi: 10.1002/acs.2561.

[63] P. Svec, A. Thakur, B. C. Shah, and S. K. Gupta, "USV Trajectory Planning for Time-Varying Motion Goals in an Environment with Obstacles," in 36th Mechanisms and Robotics Conference, Chicago, IL, USA, 2012, pp. 12971306, doi: 10.1115/detc2012-71239.

[64] I. R. Bertaska, B. Shah, V. K. Ellenrieder, P. Švec, W. Klinger, A. J. Sinisterra, and S. K. Gupta, "Experimental evaluation of automatically-generated behaviors for USV operations," Ocean Engineering, vol. 106, pp. 496-514, 2016, doi: 10.1016/j.oceaneng.2015.07.002.

[65] A. Thakur, P. Svec, S. K. Gupta, "GPU based generation of state transition models using simulations for unmanned surface vehicle trajectory planning," Robotics and Autonomous Systems, vol. 60, no. 12, pp. 1457-1471, 2012, doi: 10.1016/j.robot.2012.07.009.

[66] E. Raboin, P. Švec, D. Nau and S. K. Gupta, "Model-predictive target defense by team of unmanned surface vehicles operating in uncertain environments," 2013 IEEE International Conference on Robotics and Automation, 2013, pp. 3517-3522, doi: 10.1109/ICRA.2013.6631069.

[67] N. H. Tran, H. S. Choi, J. Y. Kim, and M. H. Lee, "Development of Rotational Motion Estimation System for a UUV/USV based on TMS320F28335 microprocessor," International Journal of Ocean System Engineering, vol. 2, no. 4, pp. 223-232, 2012, doi: 10.5574/ijose.2012.2.4.223.

[68] H. Wang, Z. Wei, C. S. Ow, K. T. Ho, B. Feng and J. Huang, "Improvement in real-time obstacle detection system for USV," 2012 12th International Conference on Control Automation Robotics \& Vision (ICARCV), 2012, pp. 1317-1322, doi: 10.1109/ICARCV.2012.6485336.

[69] G. Xia, G. Wang, X. Chen and J. Xue, "Low-cost MEMS-INS/GNSS integration using quaternion-based nonlinear filtering methods for USV," OCEANS 2016 - Shanghai, 2016, pp. 1-7, doi: 10.1109/OCEANSAP.2016.7485595.

[70] T. Temel and H. Ashrafiuon, "Sliding-mode speed controller for tracking of underactuated surface vessels with extended Kalman filter," Electronics Letters, vol. 51, no. 6, pp. 467-469, 2015, doi: 10.1049/el.2014.4516.

[71] A. J. Sinisterra, M. R. Dhanak, and K. V. Ellenrieder, "Stereovision-based target tracking system for USV operations," Ocean Engineering, vol. 133, pp. 197-214, 2017, doi: 10.1016/j.oceaneng.2017.01.024.

[72] H. Mousazadeh, et al., "Developing a navigation, guidance and obstacle avoidance algorithm for an Unmanned Surface Vehicle (USV) by algorithms fusion," Ocean Engineering, vol. 159, pp. 56-65, 2018, doi: 10.1016/j.oceaneng.2018.04.018.

[73] Li-Qiang Hou, Heng-Nian Li, Fu-Ming Huang and Pu Huang, "Tracking micro reentering USV with TDRS and ground stations using adaptive IMM method," 2011 IEEE International Conference on Information and Automation, 2011, pp. 1-7, doi: 10.1109/ICINFA.2011.5948954.

[74] N. Di Lecce, C. Laschi, M. Bibuli, G. Bruzzone and E. Zereik, "Neural dynamics and sliding mode integration for the guidance of unmanned surface vehicles," OCEANS 2015 - Genova, 2015, pp. 1-6, doi: 10.1109/OCEANSGenova.2015.7271572.

[75] Y. L. Ma, J. D. Han, and Y. Q. He, "Design of Strait-Line Tracking Controller of Under-Actuated USV Based on Back-Stepping Method and Feedback Compensation," Applied Mechanics and Materials, vol. 48-49, pp. 391-396, 2011, doi: 10.4028/www.scientific.net/amm.48-49.391.

[76] Y. L. Liao, L. Wan, and J. Y. Zhuang, "Backstepping Dynamical Sliding Mode Control Method for the Path Following of the Underactuated Surface Vessel," Procedia Engineering, 2011. https://doi.org/10.1016/j.proeng.2011.08.051

[77] X X. Chen, Z. Liu, H. Hu, L. Wang and J. Dong, "Backstepping adaptive sliding mode control for the USV course tracking system," 2017 9th International Conference on Advanced Infocomm Technology (ICAIT), 2017, pp. 265269, doi: 10.1109/ICAIT.2017.8388927. 
[78] H. Deng, R. Wang, J. Li, D. Chen, J. Sun, Y. Zhao, and J. Du, "RBF Neural Network Control for USV with Input Saturation" in 2018 2nd International Conference on Information Processing and Control Engineering, Shanghai, China, 2018, vol. 214, p. 03002, doi: 10.1051/matecconf/201821403002.

[79] T. Liu, Z. Dong, H. Du, L. Song, and Y. Mao, "Path Following Control of the Underactuated USV Based on the Improved Line-of-Sight Guidance Algorithm," Polish Maritime Research, vol. 24, no. 1, pp. 3-11, 2017, doi: 10.1515/pomr-2017-0001.

[80] E. I. Sarda, I. R. Bertaska, A. Qu and K. D. von Ellenrieder, "Development of a USV station-keeping controller," OCEANS 2015 - Genova, 2015, pp. 1-10, doi: 10.1109/OCEANS-Genova.2015.7271425.

[81] P. Švec, A. Thakur, E. Raboin, B. C. Shah, and S. K. Gupta, "Target following with motion prediction for unmanned surface vehicle operating in cluttered environments," Autonomous Robots, vol. 36, no. 4, pp. 383-405, 2013, doi: 10.1007/s10514-013-9370-z.

[82] M. Breivik, V. E. Hovstein, and T. I. Fossen, "Straight-Line Target Tracking for Unmanned Surface Vehicles Modelling," Modeling, Identification and Control (MIC), vol. 29, no. 4, pp. 131-149, 2008, doi: 10. 4173/mic.2008.4.2.

[83] P. N. F. M. Shamsuddin, M. A. Mansor, R. M. Ramli, and R. M. Q. R. Jaapar, "Development of navigation system for the unmanned surface vehicle by improving path tracking performance," Journal of Engineering Science and Technology, vol. 15, no. 2, pp. 1371-1383, 2020.

\section{BIOGRAPHIES OF AUTHORS}
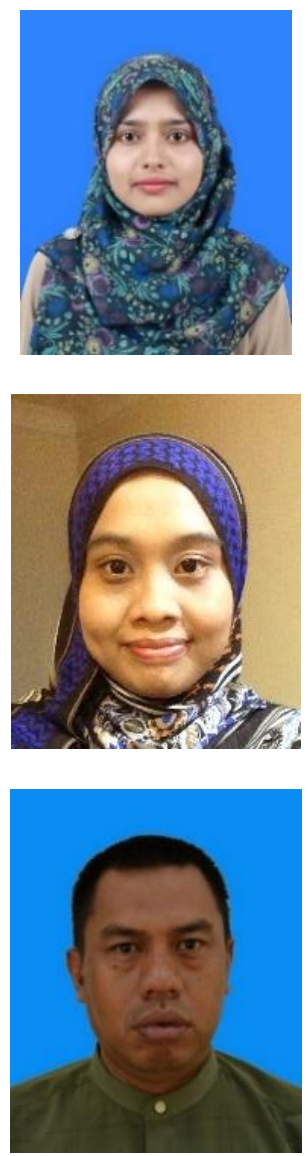

Putri Nur Farhanah Mohd Shamsuddin received her Bachelor of Engineering Technology (Electrical) degree with honor, Universiti Malaysia Pahang. She is currently further her studies in Master of Engineering Technology at Universiti Malaysia Pahang. Her research interests include the navigation system and its applications.

Roshahliza M. Ramli received her Bachelor of Engineering degree in the information system, the Master of Engineering degree in electrical engineering and computer science from the Polytechnic University of Japan, in 2010 and 2008, respectively, and the Ph.D. degree in electrical, electronic and system engineering (Signal Processing) from Universiti Kebangsaan Malaysia, in 2015. She is currently a Senior Lecturer at the Faculty of Electrical and Electronics Engineering Technology, University Malaysia Pahang. Her research interests include telecommunication technologies, digital/adaptive filtering, speech/audio processing, system and algorithm development, and their applications.

Muhamad Arifpin Bin Mansor received his Bachelor of Engineering (Electronic Engineering) at Oita University, Japan, in 1990, the Master of Engineering (Electric, Electronic, and Computer Engineering) at Kyushu Institute of Technology, in 1997, and the Doctor of Engineering (Information Science and Control Engineering), Nagaoka University of Technology, Japan, in 2010. He is currently an Associate Professor at the Faculty of Manufacturing and Mechatronic Engineering Technology, College of Engineering Technology, Universiti Malaysia Pahang, Malaysia. His research interests include Internet technology, Computer Network, Operational Research, Total Productive Maintenance, Performance Measurement, and Open Source. 